\title{
Eigenvector of gravity gradient tensor for estimating fault dips considering fault type
}

\author{
Shigekazu Kusumoto
}

\begin{abstract}
The dips of boundaries in faults and caldera walls play an important role in understanding their formation mechanisms. The fault dip is a particularly important parameter in numerical simulations for hazard map creation as the fault dip affects estimations of the area of disaster occurrence. In this study, I introduce a technique for estimating the fault dip using the eigenvector of the observed or calculated gravity gradient tensor on a profile and investigating its properties through numerical simulations. From numerical simulations, it was found that the maximum eigenvector of the tensor points to the high-density causative body, and the dip of the maximum eigenvector closely follows the dip of the normal fault. It was also found that the minimum eigenvector of the tensor points to the low-density causative body and that the dip of the minimum eigenvector closely follows the dip of the reverse fault. It was shown that the eigenvector of the gravity gradient tensor for estimating fault dips is determined by fault type. As an application of this technique, I estimated the dip of the Kurehayama Fault located in Toyama, Japan, and obtained a result that corresponded to conventional fault dip estimations by geology and geomorphology. Because the gravity gradient tensor is required for this analysis, I present a technique that estimates the gravity gradient tensor from the gravity anomaly on a profile.
\end{abstract}

Keywords: Fault dip, Gravity gradient tensor, Eigenvector, Normal fault, Reverse fault, Kurehayama Fault

\section{Introduction}

In recent years, gravity gradiometry surveys have been widely conducted to obtain detailed subsurface structure data (e.g., Jekeli 1988; Dransfield 2010; Chowdhury and Cevallos 2013; Braga et al. 2014). Data collected by these surveys is the gravity gradient tensor defined by second derivatives of the gravity potential, and its response to subsurface structures is more sensitive than the gravity anomaly. At present, gravity gradiometry surveys have mainly been performed using a helicopter. Consequently, their observation interval is about $3 \mathrm{~m}$ on the flight profile, and the observation density is very high. The gravity gradiometry surveys allowed for high observation density, high resolution, and high sensitivity to the subsurface structures; therefore, these surveys contribute greatly to the earth science and resource engineering fields in terms of being useful and powerful tools for the estimation of subsurface structures.

Correspondence: kusu@sci.u-toyama.ac.jp

Graduate School of Science and Engineering for Research (Science),

University of Toyama, 3910 Gofuku, Toyama 930-8555, Japan
Various analysis techniques using gravity gradient tensors have been suggested and discussed (e.g., Zhang et al. 2000; Beiki 2010; Martinez et al. 2013; Cevallos 2014; Li 2015). These are considered to be so-called inversion techniques. A semi-automatic interpretation method that can extract subsurface structure characteristics without geological and geophysical data input has also been developed and applied to field data (e.g., Cooper 2012; Ma 2013; Ferreira et al. 2013).

A typical semi-automatic interpretation method is an edge emphasis technique that uses extraction techniques to find locations (namely, edge) where the potential field changes abruptly due to density variations. The horizontal gravity gradient method and vertical gravity gradient method (e.g., Evjen 1936; Elkins 1951; Tsuboi and Kato 1952; Blakely and Simpson 1986) are classic edge emphasis techniques. In recent years, higher and keener extraction techniques have been suggested (e.g., Miller and Singh 1994; Cooper and Cowan 2006; Sertcelik and Kafadar 2012; Zhang et al. 2014). In addition, attention has been paid to techniques that evaluate the shape of 
the potential field (e.g., Koenderink and van Doorn 1992; Robert 2001; Zhou et al. 2013; Cevallos 2014).

Among these methodologies, a technique for estimating the dip of geological boundary using the gradient tensor of the potential fields has been developed (e.g., Beiki 2013). Beiki and Pedersen (2010) showed that the maximum eigenvector of the gravity gradient tensor points to the causative body (Fig. 1a). Since this property is common in the potential fields, Beiki (2013) applied it to a magnetic anomaly in the Assele area (Sweden) and obtained useful information on the dip of the dike swarms. Kusumoto (2015), considering that the basement consists of an aggregate of high-density prisms (Fig. 1b), applied Beiki's technique (Beiki and Pedersen 2010; Beiki 2013) to the estimation of fault dips. This method provided results wherein the fault dip estimated by the gravity gradient tensor harmonized with the dip observed from seismic surveys (Kusumoto 2015, 2016a). In addition, the dip of an earthquake source fault of the Kumamoto Earthquake that occurred in April 2016 estimated from the gravity gradient tensor also corresponded with the dip of the fault model (normal fault of $60^{\circ}$ ), thus explaining the crustal movement observed by GNSS (Global Navigation Satellite System) (Kusumoto 2016b). The range for which this method is applicable is wide from low dip to high dip (e.g., Beiki 2013; Kusumoto 2015, 2016a, 2016b), although it has some numerical instability to the vertical fault (e.g., Kusumoto 2015).

Although analyses using the gravity gradient tensor have yielded excellent results in subsurface structure estimations and edge detections, gravity gradiometry surveys have been conducted in only a few areas, limiting the tensor data available. If we were to carry out these analyses in areas where gravity gradiometry surveys have not been conducted yet, we would have to use the tensor estimated from existing gravity anomaly data.

The procedure for estimating the gravity gradient tensor from gravity anomaly data has already been suggested by Mickus and Hinojosa (2001). This technique estimates the gravity gradient tensor from spatial distribution of gravity anomalies by the Fourier transform. Since the database of gravity anomalies has been prepared, studies using the gravity gradient tensor estimated by Mickus and Hinojosa's method will progress in the future. On the other hand, it is difficult to apply this method directly to gravity anomalies obtained by gravity surveys conducted on a profile employed frequently in active fault research.

In dense gravity surveys researching fault structures in detail, profiles were set perpendicular to the fault and short-spaced gravity observations were taken along the profiles (e.g., Iwano et al. 2001; Inoue et al. 2004). It is important to find the fault shape, especially its dip, in these studies because the fault dip affects the area of disaster occurrence (e.g., Abrahamson and Somerville 1996; Takemura et al. 1998) and is an important parameter in numerical simulations for hazard map creation (e.g., Irikura and Miyake 2011). Consequently, in twodimensional gravity surveys for faults, a fault dip estimated from the eigenvectors of the gravity gradient tensor calculated from the gravity anomaly would be of additional value. In addition, since this analysis technique does not require vast calculation times, I expect it will be an effective new technique for analyzing highresolution data obtained densely, i.e., through dense gravity surveys for fault research and also airborne gravity gradiometry surveys.

In this study, I first introduce the technique for the estimation of the gravity gradient tensor from a gravity anomaly on the profile. After that, I discuss the relationship between fault dips and eigenvectors of the gravity gradient tensor and apply its result to gravity anomaly data obtained on the profile crossing the Kurehayama Fault in Toyama, Japan.

\section{Methods/Experimental}

Gravity gradient tensor on the profile

Gravity gradient tensor $\Gamma$ on the profile is defined as follows (e.g., Beiki and Pedersen 2011)
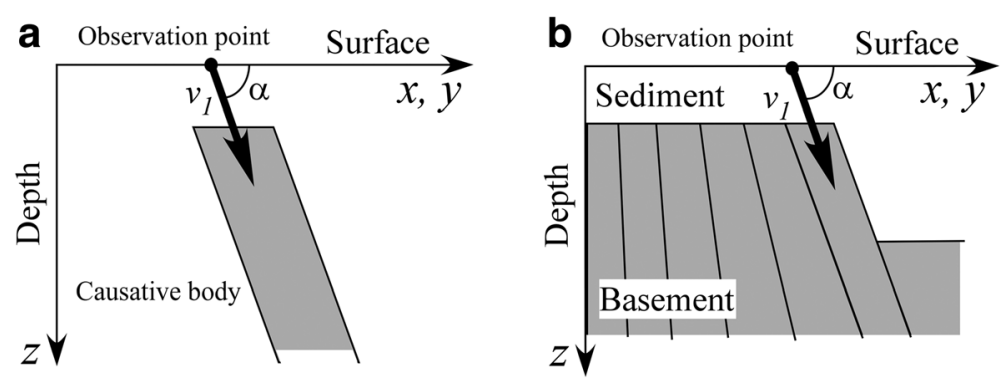

Fig. 1 Schematic illustration of the maximum eigenvectors for two-dimensional (2D) structures such as dykes and faults. a Basic model. In this figure, $v_{1}$ is the maximum eigenvector of the gravity gradient tensor and points to the causative body. The angle $a$ between the surface and the maximum eigenvector is the dip of the causative body. $\mathbf{b}$ Fault model. A basement consists of an aggregate of high-density prisms, and the angle, $a$, indicates the fault dip 


$$
\Gamma=\left[\begin{array}{ll}
g_{x x} & g_{x z} \\
g_{z x} & g_{z z}
\end{array}\right]
$$

Here, $g_{x x}, g_{x z}, g_{z x}$, and $g_{z z}$ are each component of the tensor and are defined as the first derivative of gravity vector components $g_{x}$ and $g_{z}$ for each direction. In addition, gravity vectors $g_{x}$ and $g_{z}$ are given by the first derivative of gravity potential, $W$, namely, $g_{x}=\partial W / \partial x$ and $g_{z}=\partial W / \partial z$. As the gravity potential satisfies the Laplace equation, $\partial^{2} W / \partial x^{2}+\partial^{2} W / \partial z^{2}=g_{x x}+g_{z z}=0$, we find the relationship $g_{z z}=-g_{x x}$. Also, the relationship is known to be $g_{x z}=g_{z x}$ because the gravity gradient tensor is a symmetric tensor (e.g., Torge 1989).

\section{Relationship between subsurface structure and gravity anomaly}

In the two-dimensional analyses, a structure in one direction is assumed to be infinite. Although this assumption is not realistic, it is a good approximation in fault structure analyses and gives us some practical analysis techniques. In calculations of the gravity gradient tensor from the gravity anomaly, we need gravity anomaly values at different heights. Consequently, I will show the relationship between two-dimensional subsurface structures and gravity anomalies in this subsection before estimating the gravity gradient tensor from the gravity anomaly.

As the simplest subsurface model, I set a twodimensional double layer model consisting of a sedimentary layer and a basement (Fig. 2). Horizontal positions are given by $x$, and vertical positions are given by $z$. Depth is zero $(z=0)$ on the surface, and $z$ increases with depth. As shown in Fig. 2, an average

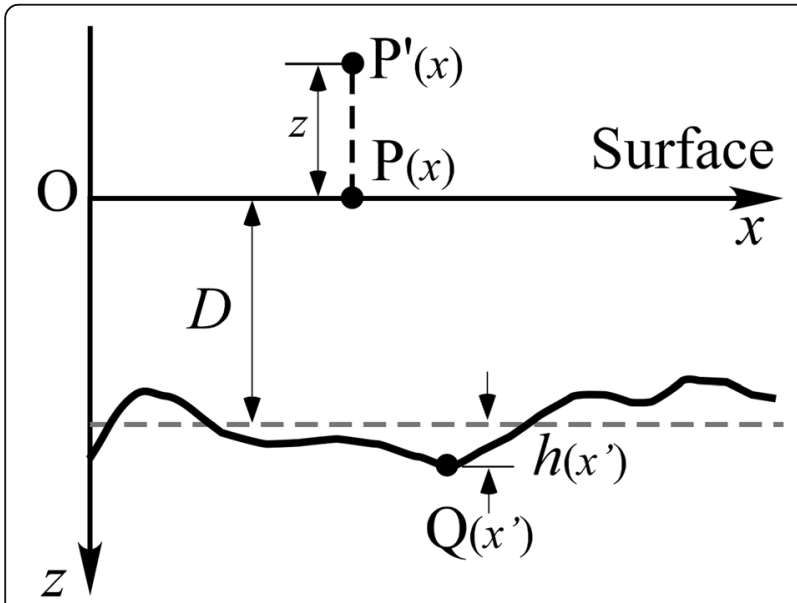

Fig. 2 Model of subsurface structure. A double-layer model consisting of a sedimentary layer and a basement is assumed here. $D$ is the average depth of the stratum boundary, and $h\left(x^{\prime}\right)$ is the deviation of the boundary from the average. Here, the deviation is assumed to be very small, i.e., $h\left(x^{\prime}\right)<<D$ boundary depth between the sedimentary layer and basement is defined as $z=D(>0)$. When the boundary surface at point $Q\left(x^{\prime}\right)$ deviates by $h\left(x^{\prime}\right)$ from the average boundary depth (Fig. 2), gravity anomaly $g_{z}(x)$ at the point $P(x)$ on the surface caused by this deviation is given by the following equation (e.g., Blakely 1996).

$$
g_{z}(x)=2 \gamma \Delta \rho \int_{-\infty}^{\infty} \int_{D}^{D+h\left(x^{\prime}\right)} \frac{z^{\prime}}{\left(x-x^{\prime}\right)^{2}+z^{\prime 2}} d x^{\prime} d z
$$

where $\gamma$ is the gravitational constant and $\Delta \rho$ is the density contrast between the sedimentary layer and basement. The integration on $z$ in Eq. (2) is solved as:

$$
\int_{D}^{D+h\left(x^{\prime}\right)} \frac{z^{\prime}}{\left(x-x^{\prime}\right)^{2}+z^{\prime 2}} d z^{\prime}=\frac{1}{2} \log \left[\frac{\left(x-x^{\prime}\right)^{2}+\left(D+h\left(x^{\prime}\right)\right)^{2}}{\left(x-x^{\prime}\right)^{2}+D^{2}}\right]
$$

here, if $h\left(x^{\prime}\right)$ is much smaller than $D$, namely, $h\left(x^{\prime}\right)<<$, $(D+h)^{2}$ is $\{D[1+(h / D)]\}^{2} \approx D^{2}(1+2 h / D)=D^{2}+2 D h$, Eq. (3) would be rewritten as follows:

$$
\begin{aligned}
\int_{D}^{D+h\left(x^{\prime}\right)} \frac{z^{\prime}}{\left(x-x^{\prime}\right)^{2}+z^{\prime 2}} d z^{\prime} & \approx \frac{1}{2} \log \left[\frac{\left(x-x^{\prime}\right)^{2}+D^{2}+2 D h\left(x^{\prime}\right)}{\left(x-x^{\prime}\right)^{2}+D^{2}}\right] \\
& =\frac{1}{2} \log \left[1+\frac{2 D h\left(x^{\prime}\right)}{\left(x-x^{\prime}\right)^{2}+D^{2}}\right]
\end{aligned}
$$

In general, if $-1<\xi \leq 1$ in $\log (1+\xi)$, we have the following approximation (e.g., Gradshteyn and Ryzhik 2007)

$$
\begin{aligned}
\log (1+\xi) & =\xi-\frac{1}{2} \xi^{2}+\frac{1}{3} \xi^{3}-\frac{1}{4} \xi^{4}+\cdots \\
& =\sum_{p=1}^{\infty}(-1)^{p+1} \frac{\xi^{p}}{p}
\end{aligned}
$$

The second term, $2 D h /\left[\left(x-x^{\prime}\right)^{2}+D^{2}\right]$, in Eq. (4) is small because $D>>h$. We can use Eq. (5) to derive a linear approximate equation of Eq. (4). By neglecting higher terms of $\xi$, Eq. (3) or (4) is rewritten as follows:

$$
\int_{D}^{D+h\left(x^{\prime}\right)} \frac{z^{\prime}}{\left(x-x^{\prime}\right)^{2}+z^{\prime 2}} d z^{\prime} \approx \frac{D h\left(x^{\prime}\right)}{\left(x-x^{\prime}\right)^{2}+D^{2}}
$$

Consequently, we obtained the following equation.

$$
g_{z}(x) \approx 2 \gamma D \Delta \rho \int_{-\infty}^{\infty} \frac{h\left(x^{\prime}\right)}{\left(x-x^{\prime}\right)^{2}+D^{2}} d x
$$

Here, I introduce a new function, $\phi$, defined by:

$$
\phi(x)=\frac{1}{x^{2}+D^{2}}
$$

and Eq. (7) is rewritten as follows: 


$$
g_{z}(x)=2 \gamma D \Delta \rho \int_{-\infty}^{\infty} \phi\left(x-x^{\prime}\right) h\left(x^{\prime}\right) d x^{\prime}
$$

This form is convoluted, and we obtain Eq. (10) by applying the Fourier transformation to Eq. (9)

$$
G_{z}=2 \gamma D \Delta \rho \Phi H
$$

where, $G_{z}, \Phi$, and $H$ are Fourier transforms of $g_{z}(x)$, $\phi(x)$, and $h(x)$, respectively. As is well known, the Fourier transform of Eq. (8) is (e.g., Blakely 1996; Gradshteyn and Ryzhik 2007)

$$
\Phi=\frac{\pi}{D} e^{-D|k|}
$$

Here, $|k|=i k_{z}=\left|k_{x}\right|$ (e.g., Blakely 1996) and $k_{x}$ is the wave number in the $x$ direction. Here, I employed the Fourier transform, $F$, of a function $f(x)$ defined as follows (e.g., Blakely 1996):

$$
F=\int_{-\infty}^{\infty} f(x) e^{-i k x} d x
$$

By Eq. (11), Eq. (10) is rewritten as:

$$
G_{z}=2 \pi \gamma \Delta \rho H e^{-D|k|}
$$

This is the relationship between gravity anomaly on the profile and two-dimensional subsurface structure.

\section{Relationship between gravity anomaly and gravity gradient tensor}

As shown in the previous section, the gravity gradient tensor is given by the second derivative of the gravity potential. The relationship between gravity anomaly $g_{z}$ and gravity potential $W$ is

$$
W=-\int g_{z} d z
$$

From Eq. (13), the equation giving the gravity anomaly at point $P^{\prime}(x)$ of an arbitrary height $z$ from the surface (Fig. 2) is obtained in the Fourier domain as follows:

$$
G_{z}=2 \pi \gamma \Delta \rho H e^{-(D+z)|k|}
$$

By integrating this equation to $z$ and substituting $z=0$, we obtain the gravity potential at the surface. If the Fourier transform of the gravitational potential is represented by $U$, from these calculations, the $U$ would be given by $G_{z}$ as follows:

$$
U=\frac{1}{|k|} G_{z}
$$

As the $x$ direction component of gravity anomaly is given by the first derivative in the $x$ direction of the gravity potential $W$, the $g_{x}$ in the Fourier domain, $G_{x}$, would be given by a differential formula in the Fourier domain (e.g., Blakely 1996) as follows:

$$
G_{x}=i k_{x} U
$$

From Eq. (16), we obtained

$$
G_{x}=\frac{i k_{x}}{|k|} G_{z}
$$

$g_{x x}$ in the Fourier domain is given by

$$
G_{x x}=\frac{-k_{x}^{2}}{|k|} G_{z}
$$

We can obtain $g_{x x}$ by applying the inverse Fourier transform to $G_{x x}$, and $g_{z z}$ would be obtained from the relationship of $g_{z z}=-g_{x x}$. The other component $g_{z x}\left(=g_{x z}\right)$ would be given by:

$$
G_{z x}=G_{x z}=i k_{x} G_{z}
$$

where $G_{z x}$ and $G_{x z}$ are the Fourier transform of $g_{z x}$ and $g_{x z}$.

Here, although I showed a technique to calculate the gravity gradient tensor in the Fourier domain, there is another technique to calculate the tensor by a simple finite-difference method (e.g., Blakely 1996) of gravity vectors $g_{x}$ and $g_{z}$ in the space domain.

\section{Relationship between subsurface structures and eigenvectors}

As indicated by Beiki and Pedersen (2010), the maximum eigenvector of the gravity gradient tensor points to the causative body of the gravity anomaly (Fig. 1a). They also pointed out that the minimum eigenvector of the tensor indicates the strike direction of structures such as dikes in three-dimensional analyses. Since there are two perpendicular eigenvectors of the gravity gradient tensor in the two-dimensional analyses, it is expected that the minimum eigenvector of the tensor will point to the low-density causative body or medium if the maximum eigenvector of the tensor points out highdensity causative bodies such as a dike in a low-density layer such as a sedimentary layer.

To clear this inference, I calculated the gravity gradient tensor on the profile caused by the model shown in Fig. 3 and investigated the dips of the maximum and minimum eigenvectors of the tensor. The model shown in Fig. 3 has a width and height of 0.25 and $2.0 \mathrm{~km}$, respectively.

Each component of the gravity gradient tensor caused by two-dimensional structures such as the dike shown in Fig. 3 is given by Telford et al. (1990). The relationship between eigenvectors and structural boundaries will be discussed widely in this study; I therefore employed calculation formulas given by Talwani et al. (1959). Talwani et al. (1959) show well-known calculation formulas giving $g_{x}$ and $g_{z}$ for two-dimensional arbitrary structures 


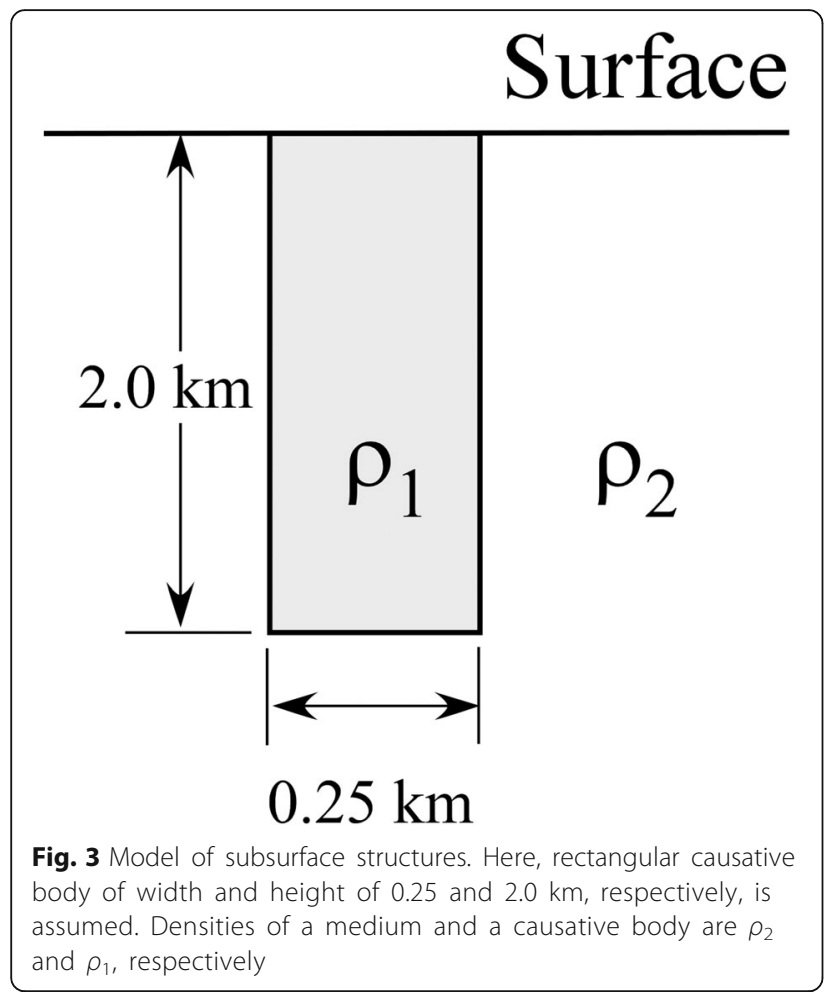

closed by a polygon. In this study, I obtained $g_{z x}\left(=g_{x z}\right)$ and $g_{x x}$ components by the numerical differentiation of $g_{z}$ and $g_{x}$, and the $g_{z z}$ component was given by $g_{z z}=-g_{x x}$. A simple finite-difference method (e.g., Blakely 1996) was employed for these numerical differentiations. In addition, the dip of each eigenvector $(\alpha)$ was calculated by

$$
\alpha=\arctan \left(\frac{v_{z}}{v_{x}}\right)
$$

where $v_{x}$ and $v_{z}$ are $x$ and $z$ components of each eigenvector.

\section{Results and discussion}

\section{Density structures and eigenvectors}

Figure 4a shows distributions of the maximum (red) and minimum (blue) eigenvectors of the gravity gradient tensor caused by the model structure (Fig. 3) whose density contrast $\left(\Delta \rho=\rho_{1}-\rho_{2}\right)$ is $200 \mathrm{~kg} / \mathrm{m}^{3}$. Figure $4 \mathrm{~b}$ shows distributions of the maximum (red) and minimum (blue) eigenvectors of the tensor caused by the model structure (Fig. 3) whose density contrast $(\Delta \rho)$ is $-200 \mathrm{~kg} / \mathrm{m}^{3}$. In each figure, the lengths of all the eigenvectors are the same.

From Fig. 4a, it is found that the maximum eigenvector of the gravity gradient tensor points to a high-density causative body if the body is embedded in the low-density medium. In this case, the minimum eigenvector of the tensor points to the low-density medium around the high-density body. On the other hand, the minimum

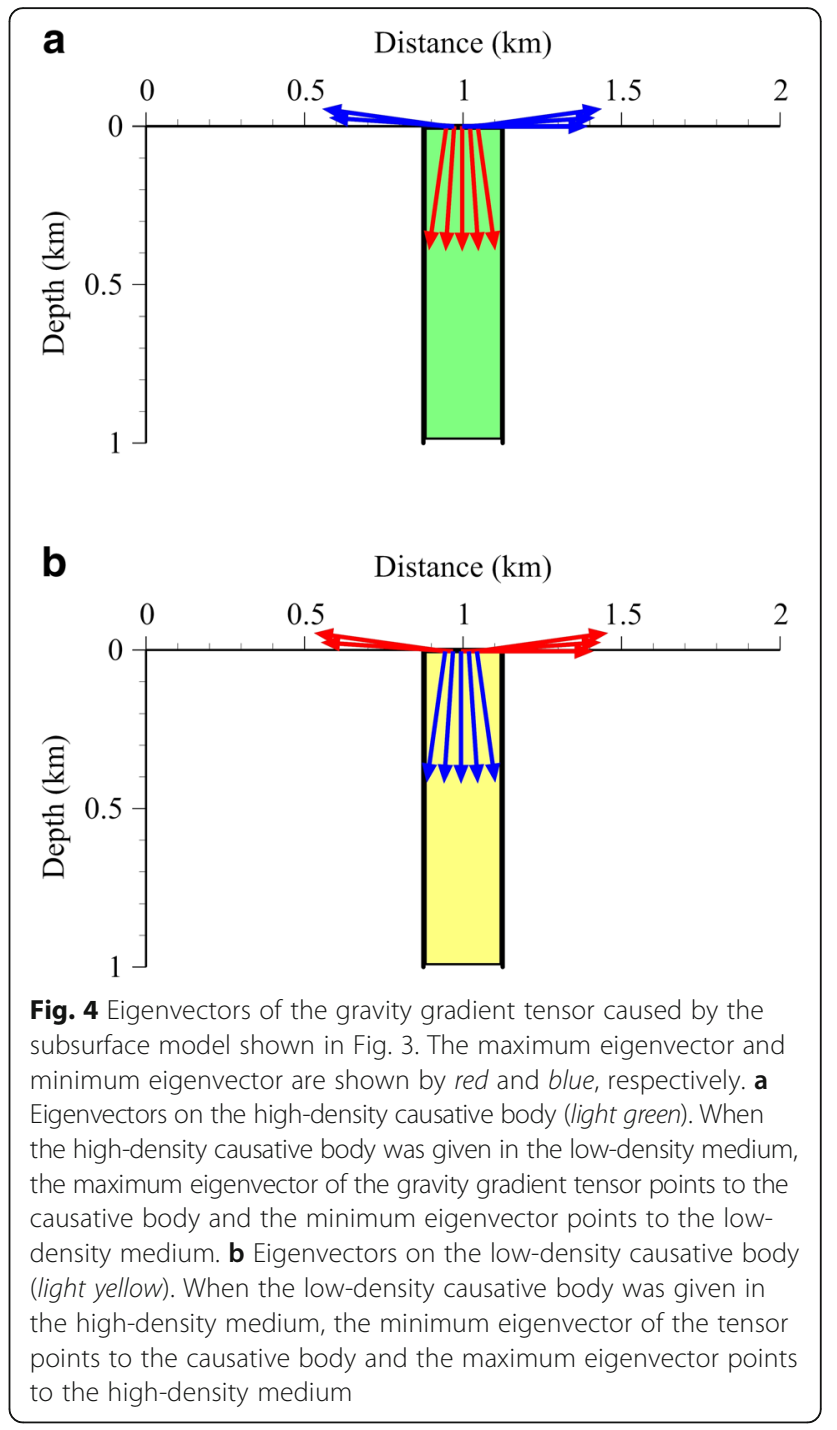

eigenvector of the gravity gradient tensor points to a low-density causative body if the body is embedded in the high-density medium. In this case, the maximum eigenvector of the tensor points to the high-density medium around the low-density body. From these results, in the two-dimensional analyses, it was shown that the maximum eigenvector points to a highdensity causative body and the minimum eigenvectors points to a low-density causative body.

In Fig. 4, there are vectors pointing to the area $z<0$. This indicates that $\alpha$ is negative. Structures exist underground, and the negative $\alpha$ is not realistic. Consequently, I will add $\pi$ to $\alpha$ if $\alpha$ is negative.

\section{Fault types and eigenvectors}

In calderas and/or sedimentary basins, high-density and low-density materials are in contact with each other via normal faults and/or reverse faults. In gravity anomalies and gravity gradient tensors, differences in fault type are 
defined as differences in density structure. As it was shown that the behavior of each eigenvector is dependent on the density structure in the previous subsection, I investigated the relationship between eigenvectors and fault type by the simplified sedimentary basin models.

Figure $5 \mathrm{a}$ is a simplified sedimentary basin model in which the sedimentary layer is in contact with the basement by normal faults, and Fig. 5b is a simplified sedimentary basin model in which the sedimentary layer is in contact with the basement by reverse faults. Density contrast between sedimentary layer and basement is assumed to be $-200 \mathrm{~kg} / \mathrm{m}^{3}$.

In Fig. 6, I showed distributions of the maximum (red) and minimum (blue) eigenvectors of the gravity gradient tensor caused by these models. In each figure, the lengths of all the eigenvectors are the same, because we are interested in the fault dip and only angle information is necessary for this study.

From Fig. 6a, it is found that the dip of the maximum eigenvector of the gravity gradient tensor closely follows the dip of the normal fault. When the basement distributes near the surface, the maximum eigenvector points in the vertical direction to the high-density basement. The effect of the high-density basement is weak in the sedimentary layer area, while the effect of the lowdensity sedimentary layer is strong; therefore, the minimum eigenvector points in the vertical direction to the low-density sediment and the maximum eigenvector points in the horizontal direction.

When the boundary is a reverse fault, from Fig. 6b, it is found that the dip of the minimum eigenvector of the gravity gradient tensor indicates the dip of the fault well.

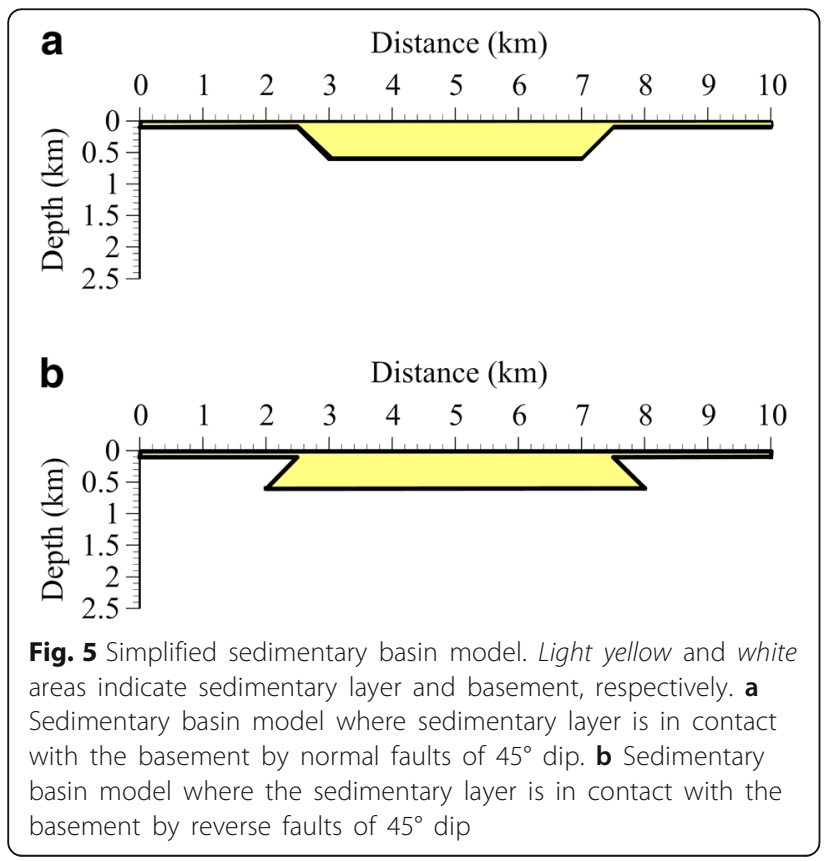

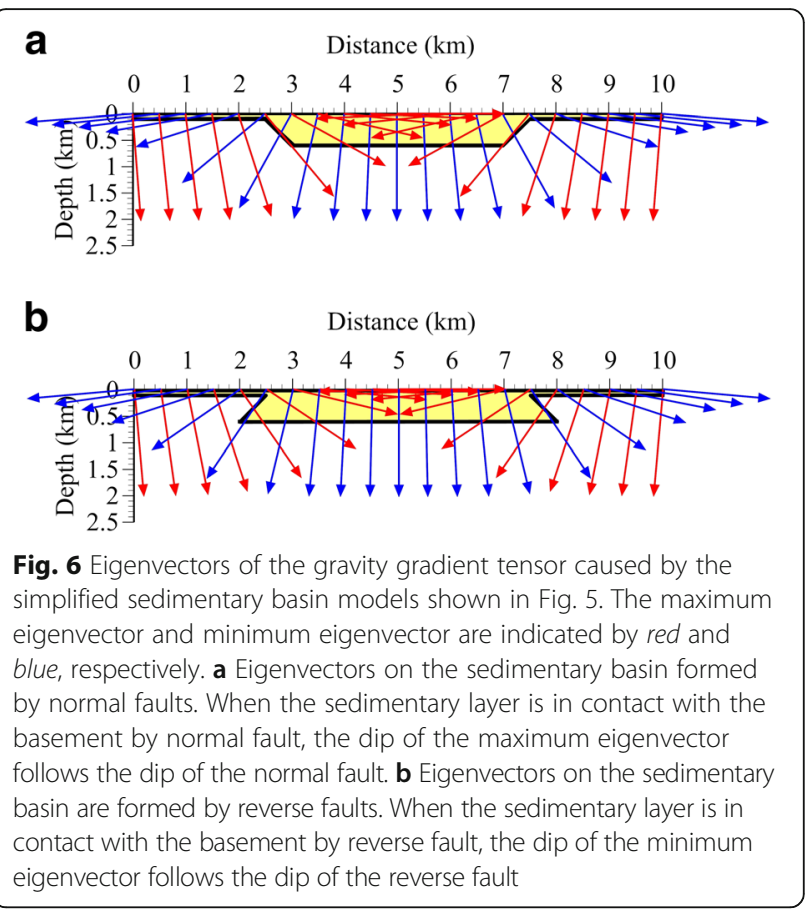

The maximum eigenvector on the basement points in the vertical direction to the high-density basement, and the minimum eigenvector points in the horizontal direction. Since the low-density sediment distributes near the surface in the sedimentary layer area, the minimum eigenvector points vertically.

From these results, it was concluded that if the structural boundary is a normal fault, its dip can be estimated from the dip of the maximum eigenvector of the gravity gradient tensor, and if the boundary is a reverse fault, its dip can be estimated from the dip of the minimum eigenvector of the tensor. In addition, in the area away from the boundary, it was found that the maximum eigenvector on the basement and the minimum eigenvector on the sediment point in the vertical direction, and the maximum eigenvector on the sediment and the minimum eigenvector on the basement point in the horizontal direction, regardless of whether the boundary is a normal fault or reverse fault.

\section{Subsurface structures and eigenvectors}

By simple numerical simulations, it was found that the maximum eigenvector of the gravity gradient tensor points to a high-density causative body and that the minimum eigenvector points to a low-density causative body. In addition, it was found that the dip of the maximum eigenvector of the tensor closely follows the dip of the normal fault and that the dip of the minimum eigenvector closely follows the dip of the reverse fault. 
As mentioned above, Beiki and Pedersen (2010) have already pointed out that the maximum eigenvector of the gravity gradient tensor points to the high-density causative body. The result in Fig. 4a confirms that their results are true for the two-dimensional analyses as well. When the basement distributes near the surface, the maximum eigenvector points in the vertical direction. This property also shows that Beiki and Pedersen (2010) are correct, and the idea of the basement as an aggregate of high-density prisms (Fig. 1b), suggested by Kusumoto (2015, 2016b), would not be incorrect.

As to why the dip of normal fault was given by the dip of the maximum eigenvector of the gravity gradient tensor, I considered that the lower part of the boundary structure (fault) exists inside the low-density area more than its upper part. Therefore, because the gravity gradient tensor is most sensitive to the subsurface structures near the surface, the structure shown in Fig. 5a was considered a high-density body that intruded into the low-density layer, and the dip of the normal fault was given by the dip of the maximum eigenvector. I believe Kusumoto (2015, 2016a, 2016b) was able to obtain results that coincided with seismic surveys since he estimated the fault dip in normal fault regions by the maximum eigenvector of the tensor.

On the other hand, when the maximum eigenvector points to high-density causative bodies embedded in low-density medium or low-density causative bodies embedded in a high-density medium, the minimum eigenvector points to the low-density mediums or to the causative bodies. Beiki and Pedersen (2010) have not explicitly referred to analyses of low-density causative bodies using eigenvectors. Since it is necessary to analyze anomalies caused by low-density bodies in the field, it seems that the result, in which the minimum eigenvector points to the low-density bodies, would play an important role in subsurface structure estimation, although this is the result of two-dimensional analysis.

In addition, it was found that the dip of the minimum eigenvector of the gravity gradient tensor gave the dip of the reverse fault. As to the reason why the dip of reverse fault was given by the minimum eigenvector of the gravity gradient tensor, I considered that the lower part of the boundary structure (fault) exists inside the highdensity area more than its upper part. Namely, because this structure was considered a low-density body that intruded into the high-density layer, the dip of the reverse fault was given by the dip of the minimum eigenvector of the gravity gradient tensor.

As is understood from the results and discussions obtained in this study, selecting a suitable eigenvector for estimating the fault dip is important. If the study area is not too wide and prior geological information is available, the eigenvector that should be employed for estimating the fault dip correctly would be selected based on the information. If the study area was a fault area where normal faults were mainly distributed, the maximum eigenvector of the gravity gradient tensor would be employed for estimating the fault dip. If the study area was a fault area where reverse faults were mainly distributed, the minimum eigenvector would be employed.

In the three-dimensional study for high-density causative bodies, it is pointed out that the minimum eigenvector is parallel to the strike direction of the structure (Beiki and Pedersen 2010; Beiki 2013). However, in the two-dimensional analyses, the strike direction of the structure is perpendicular to $x$ - and $z$-axes and does not appear in the analyses. As it is difficult to directly compare the properties of the minimum eigenvector obtained in different dimensions, in the future, it would be necessary to discuss detailed properties of the minimum eigenvector.

\section{Application to field data}

As an application of the techniques, I estimated the dip of the Kurehayama Fault located in Toyama, Japan. The Kurehayama Fault is a reverse fault located at the center of the Toyama basin, and it strikes in the NNE-SSW direction (Fig. 7). The length of the fault is about $22 \mathrm{~km}$, and the fault dip is about $45^{\circ}$ (e.g., The Headquarters for Earthquake Research Promotion 2008; Toyama City 2013). The Toyama City has carried out seismic surveys and dense gravity surveys crossing this fault (Toyama City 2013). Toyama City (2013) set three profiles crossing the Kurehayama Fault, and the dense gravity surveys of $50 \mathrm{~m}$ spaced measurements have been conducted on these profiles, although spacing of several hundred meters has been usually employed for these surveys. Here, I used gravity anomaly data on the profile located at the shoreline. Figure 8 shows the Bouguer anomaly in which the Bouguer density of $2260 \mathrm{~kg} / \mathrm{m}^{3}$ was assumed (Toyama City 2013). The indication "Kurehayama Fault" shown in this figure indicates a rough fault location.

I applied the techniques to the Bouguer anomaly and obtained the gravity gradient tensor shown in Fig. 9. Figure 10 shows distributions of the maximum eigenvector (red) and the minimum eigenvector (blue) of the gravity gradient tensor. Since the Kurehayama Fault is a reverse fault, I focus on the dip of the minimum eigenvector. From Fig. 10, it is found that the dip $(\alpha)$ of the Kurehayama Fault was about $138^{\circ}$. Since the angle $\alpha$ is measured clockwise from the surface $(x$-axis), it seems that the obtained dip indicates the dip of the reverse fault of $42^{\circ}$. This fault dip is consistent with conventional data. 


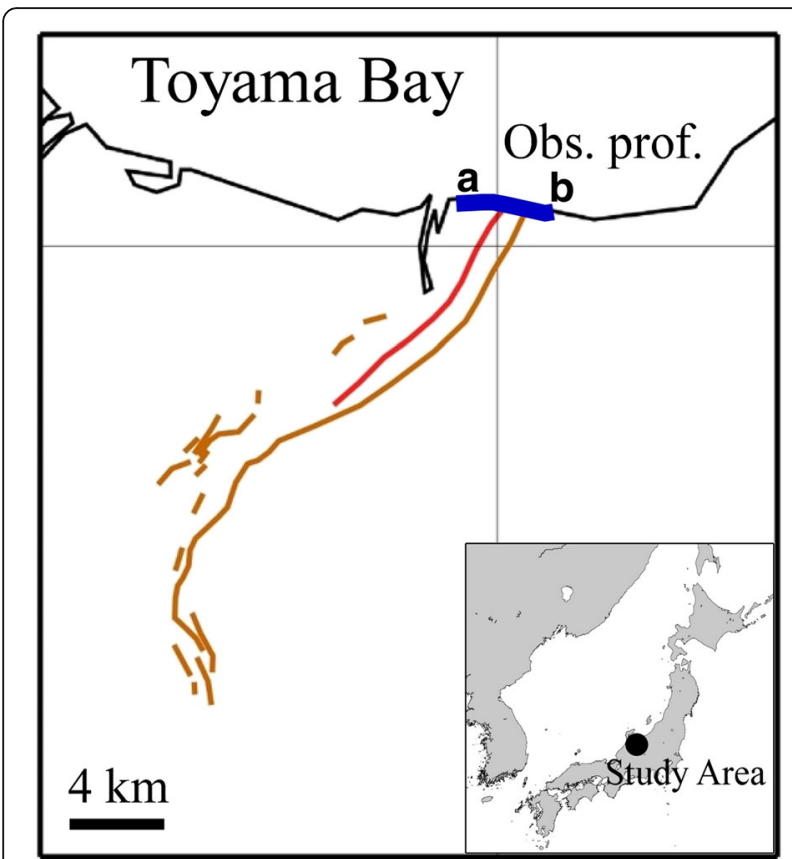

Fig. 7 Location map of the study area. Kurehayama Fault is a reverse fault located in the center of the Toyama Basin, Toyama Prefecture, Japan. Its location has been estimated by topographic, geological, and geophysical data. The red line and brown lines denote the estimated location of the Kurehayama Fault, Toyama City (Toyama City 2013), and The Headquarters for Earthquake Research Promotion (The Headquarters for Earthquake Research Promotion 2008), respectively. Blue line $a-b$ indicates the dense gravity survey profile, which has gravity observation points at about $50 \mathrm{~m}$ intervals

The estimated fault dip would be the dip near the surface because the method employs the gravity gradient tensor, which is sensitive to subsurface structures near the surface. Since it is important to know quantitatively which depth the estimated fault dip is, in the future, it would be necessary to develop a technique

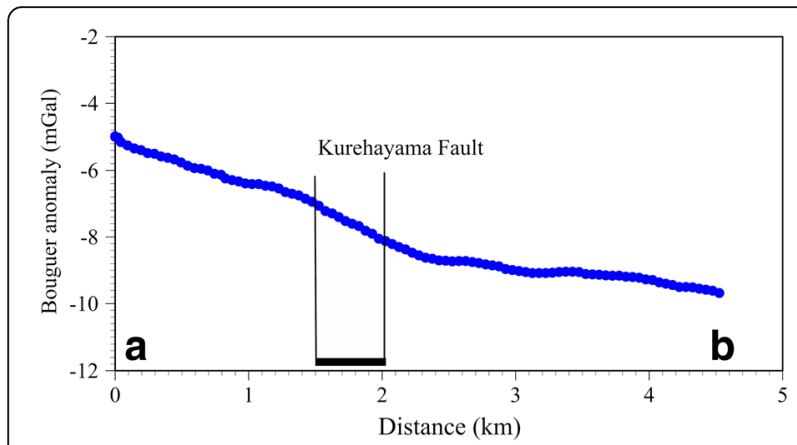

Fig. 8 Bouguer anomalies on the profiles (after Toyama City 2013). The Bouguer density of $2260 \mathrm{~kg} / \mathrm{m}^{3}$ is assumed. The "Kurehayama Fault" shown in this figure indicates a rough fault location by Toyama City (Toyama City 2013). The unit of the gravity anomaly is given in milligal, and $\mathrm{mGal}=10^{-5} \mathrm{~m} / \mathrm{s}^{2}$

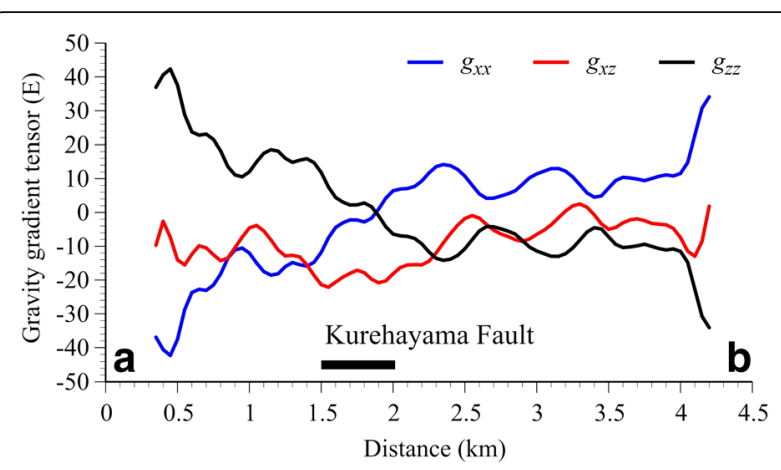

Fig. 9 Gravity gradient tensor $\left(g_{x x}, g_{x z}\left(=g_{z x}\right), g_{z z}\right)$ on the profile. These are estimated from the Bouguer anomalies on the profile shown in Fig. 8. The component of $g_{x x}$ and $g_{x z}$ is calculated by a finite-difference method of gravity vectors $g_{x}$ and $g_{z}$ in the space domain. The "Kurehayama Fault" shown in this figure indicates a rough fault location by Toyama City (Toyama City 2013). The unit of the gravity gradient tensor is given in $E$ (Ë̈tvös), and $1 E=0.1 \mathrm{mGal} / \mathrm{km}$

estimating the depth of the estimated dip or the dip in the arbitrary depth.

\section{Conclusions}

In this study, I showed techniques for estimating the gravity gradient tensor from gravity anomalies on the profile and for estimating the fault dip by eigenvector of

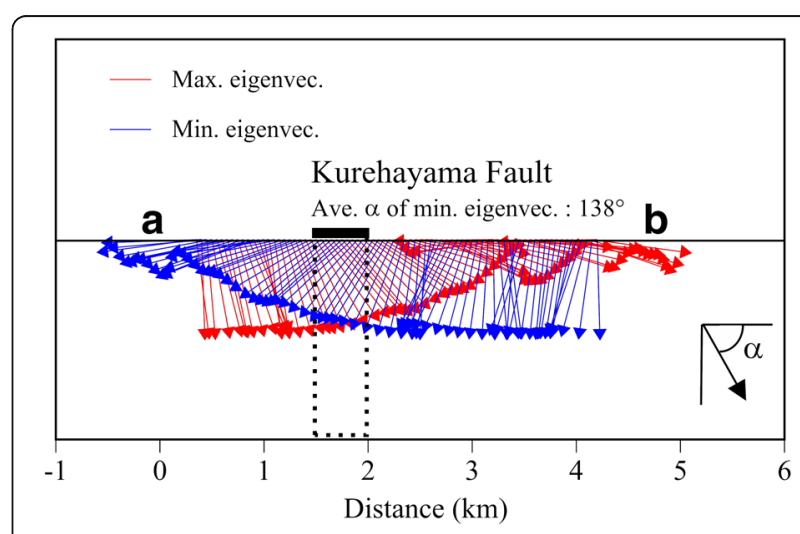

Fig. 10 Eigenvectors of the gravity gradient tensor on the profile shown in Fig. 7. The maximum eigenvector and minimum eigenvector are indicated by red and blue, respectively. The dips of eigenvectors are given clockwise from $x$-axis to $z$-axis. Since it is known that the Kurehayama Fault is a reverse fault, we focus on the minimum eigenvector of the tensor. The "Kurehayama Fault" shown in this figure indicates a tentative fault location in Toyama City (Toyama City 2013). The average dip of the minimum eigenvector in the Kurehayama Fault zone shown by a rectangle with dashed lines is about $138^{\circ}$, and this angle indicates that the Kurehayama Fault would be a reverse fault of $42^{\circ}$. In addition, the maximum eigenvectors on the right side of the "Kurehayama Fault" shown in this figure point to the vertical direction, and the minimum eigenvectors in the left side of the "Kurehayama Fault" point to the vertical direction 
the observed or calculated gravity gradient tensor on the profile. I also investigated its properties by numerical simulations.

From numerical simulations, it was found that the maximum eigenvector of the tensor points to a highdensity causative body and that the dip of the maximum eigenvector closely follows the dip of the normal fault. In addition, if the basement distributes near the surface, the maximum eigenvector points to the vertical direction. They have been pointed out already in previous studies, and the results shown in here confirmed that their results are true for the two-dimensional analyses as well. On the other hand, it was found that the minimum eigenvector of the tensor points to a low-density causative body and that the dip of the minimum eigenvector closely follows the dip of the reverse fault. Since eigenvector analyses of the anomalies caused by the lowdensity causative body have not been discussed explicitly in previous studies, these results would play an important role in estimations of subsurface structures in the future. From these results, it was found that the eigenvector of the gravity gradient tensor for estimating fault dips is determined by fault type, and we would estimate the fault dip correctly if we were to employ suitable eigenvectors based on prior information.

As an application of suggestions, I estimated the dip of the Kurehayama Fault located in Toyama, Japan, and obtained the fault dip of about $42^{\circ}$ as the dip of the minimum eigenvector of the gravity gradient tensor because the fault is the reverse fault. This dip harmonized with conventional geological information.

Since the analysis technique shown in this study does not require complex calculations and vast calculation times, it will be an effective technique for analyzing high-resolution data obtained densely by not only dense gravity surveys for fault research but also airborne gravity or gravity gradiometry surveys.

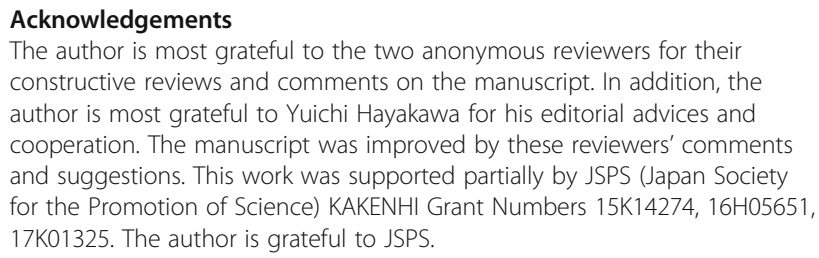
constructive reviews and comments on the manuscript. In addition, the author is most grateful to Yuichi Hayakawa for his editorial advices and cooperation. The manuscript was improved by these reviewers' comments and suggestions. This work was supported partially by JSPS (Japan Society for the Promotion of Science) KAKENHI Grant Numbers 15K14274, 16H05651, 17K01325. The author is grateful to JSPS.

\section{Funding}

This work was supported partially by JSPS KAKENHI Grant Numbers 15K14274, 16H05651, and 17K01325.

\section{Author's contributions}

SK planned this study and conducted all the calculations and discussion. He also drafted this manuscript.

Authors' information

SK is an associate professor at the University of Toyama.

\section{Competing interests}

The author declares no competing interests.

\section{Publisher's Note}

Springer Nature remains neutral with regard to jurisdictional claims in published maps and institutional affiliations.

Received: 30 November 2016 Accepted: 24 May 2017

Published online: 02 June 2017

\section{References}

Abrahamson NA, Somerville P (1996) Effects of the hanging wall and footwall on ground motions recorded during the Northridge earthquake. Bull Seism Soc Am 86:593-\$99

Beiki M (2010) Analytic signals of gravity gradient tensor and their application to estimate source location. Geophysics 75:159-174

Beiki M (2013) TSVD analysis of Euler deconvolution to improve estimating magnetic source parameters: an example from the Asele area, Sweden. J Appl Geophys 90:82-91

Beiki M, Pedersen LB (2010) Eigenvector analysis of gravity gradient tensor to locate geologic bodies. Geophysics 75:137-149

Beiki M, Pedersen LB (2011) Window constrained inversion of gravity gradient tensor data using dike and contact models. Geophysics 76:159-170

Blakely RJ (1996) Potential theory in gravity and magnetic applications. Cambridge University Press, Cambridge

Blakely R, Simpson RW (1986) Approximating edges of source bodies from magnetic or gravity anomalies. Geophysics 51:1494-1498

Braga MA, Endo I, Galbiatti HF, Carlos DU (2014) 3D full tensor gradiometry and Falcon systems data analysis for iron ore exploration: Bau Mine, Quadrilatero Ferrifero, Minas Gerais, Brazil. Geophysics 79:B213-B220

Cevallos C (2014) Automatic generation of 3D geophysical models using curvatures derived from airborne gravity gradient data. Geophysics 79:G49-G58

Chowdhury PR, Cevallos C (2013) Geometric shapes derived from airborne gravity gradiometry data: new tools for the explorationist. Lead Edge 32:1468-1474

Cooper GRJ (2012) The removal of unwanted edge contours from gravity datasets. Expl Geophys 44:42-47

Cooper GRJ, Cowan DR (2006) Enhancing potential field data using filters based on the local phase. Comp Geosci 32:1585-1591

Dransfield M (2010) Conforing Falcon gravity and the global gravity anomaly. Geophys Prospect 58:469-483

Elkins TA (1951) The second derivative method of gravity interpretation. Geophysics 16:29-50

Evjen HM (1936) The place of the vertical gradient in gravitational interpretations. Geophysics 1:127-137

Ferreira FJF, de Souza J, de B e S Bongiolo A, de Castro LG (2013) Enhancement of the total horizontal gradient of magnetic anomalies using the tilt angle. Geophysics 78:J33-J41

Gradshteyn IS, Ryzhik IM (2007) Table of integrals, series, and products, 7th edn. Academic press Elsevier, Oxford

Inoue N, Tanaka Y, Itoh H, Iwano S, Kitada N, Fukuda Y, Takemura K (2004) Density of sediment in Kyoto basin inferred from 2D gravity analysis along Horikawa-Oguraike and Kuzebashi seismic survey lines. Zisin 2(57):45-54 (in Japanese with English abstract)

Irikura K, Miyake H (2011) Recipe for predicting strong ground motion from crustal earthquake scenarios. Pure Appl Geophys 168:85-104. doi:10.1007/ s00024-010-0150-9

Iwano S, Fukuda Y, Ishiyama T (2001) An estimation of fault related structures by means of one-dimensional gravity surveys - case studies at the Katagihara Fault and the Fumotomura Fault. J Geog 110:44-57 (in Japanese with English abstract)

Jekeli C (1988) The gravity gradiometer survey system (GGSS). EOS Trans AGU 69:105

Koenderink JJ, van Doorn AJ (1992) Surface shape and curvature scales. Im Vis Comp 10:557-564

Kusumoto S (2015) Estimation of dip angle of fault or structural boundary by eigenvectors of gravity gradient tensors. Butsuri-Tansa 68:277-287 (in Japanese with English abstract)

Kusumoto S (2016a) Structural analysis of caldera and buried caldera by semiautomatic interpretation techniques using gravity gradient tensor: a case study in central Kyushu Japan. In: Nemeth K (ed) Updates in Volcanology From volcano modelling to volcano geology. InTech, Rijeka 
Kusumoto S (2016b) Dip distribution of Oita-Kumamoto Tectonic Line located in central Kyushu, Japan, estimated by eigenvectors of gravity gradient tensor. Earth Plan Space 68:153. doi:10.1186/s40623-016-0529-7

Li X (2015) Curvature of a geometric surface and curvature of gravity and magnetic anomalies. Geophysics 80:G15-G26

Ma G (2013) Edge detection of potential field data using improved local phase filter. Expl Geophys 44:36-41

Martinez C, Li Y, Krahenbuhl R, Braga MA (2013) 3D inversion of airborne gravity gradiometry data in mineral exploration: a case study in the Quadrilatero Ferrifero, Brazil. Geophysics 78:B1-B11

Mickus KL, Hinojosa JH (2001) The complete gravity gradient tensor derived from the vertical component of gravity: a Fourier transform technique. J Appl Geophys 46:159-174

Miller HG, Singh V (1994) Potential field tilt-a new concept for location of potential field sources. J Appl Geophys 32:213-217

Robert A (2001) Curvature attributes and their application to 3D interpreted horizons. First Break 19:85-99

Sertcelik I, Kafadar O (2012) Application of edge detection to potential field data using eigenvalue analysis of structure tensor. J Appl Geophys 84:86-94

Takemura M, Moroi T, Yashiro K (1998) Characteristics of strong ground motions as deduced from spatial distributions of damages due to the destructive inland earthquakes from 1891 to 1995 in Japan. Zisin 2(50):485-505 (in Japanese with English abstract)

Talwani M, Lamar WJ, Landisman M (1959) Rapid gravity computations for twodimensional bodies with application to the Mendocino submarine fracture zone. J Geophys Res 64:49-59

Telford WM, Geldart LP, Sheriff RE (1990) Applied geophysics. Cambridge University Press, Cambridge

The Headquarters for Earthquake Research Promotion (2008) Evaluations of Tonami Fault zone and Kurehayama Fault zone. In: The Headquarters for Earthquake Research Promotion web site. http://www.jishin.go.jp/main/ chousa/katsudansou_pdf/56_tonami_kureha_2.pdf (in Japanese). Accessed 21 Nov 2016

Torge W (1989) Gravimetry. Walter de Gruyter, Berlin

Toyama City (2013) Research report on Kurehayama Fault (2). Toyama-shi, Toyama (in Japanese)

Tsuboi C, Kato M (1952) The first and second vertical derivatives of gravity. J Phys Earth 1:95-96

Zhang C, Mushayandebvu MF, Reid AB, Fairhead JD, Odegrad ME (2000) Euler deconvolution of gravity tensor gradient data. Geophysics 65:512-520

Zhang X, Yu P, Tang R, Xiang Y, Zhao C-J (2014) Edge enhancement of potential field data using an enhanced tilt angle. Expl Geophys 46:276-283. doi:10. 1071/EG13104

Zhou W, Du X, Li J (2013) The limitation of curvature gravity gradient tensor for edge detection and a method for overcoming it. J Appl Geophys 98:237-242

\section{Submit your manuscript to a SpringerOpen ${ }^{\circ}$ journal and benefit from:}

- Convenient online submission

- Rigorous peer review

- Open access: articles freely available online

- High visibility within the field

Retaining the copyright to your article

Submit your next manuscript at $>$ springeropen.com 